\title{
The information from muon arrival time distributions of high-energy EAS as measured with the KASCADE detector
}

\author{
KASCADE Collaboration
}

\author{
T. Antoni ${ }^{\mathrm{a}}$, W.D. Apel ${ }^{\mathrm{b}}$, A.F. Badea ${ }^{\mathrm{a}, 1,2}$, K. Bekk $^{\mathrm{b}}$, \\ A. Bercuci ${ }^{b}, 2$, H. Blümer ${ }^{b, a}$, H. Bozdog ${ }^{b}$, I.M. Brancus ${ }^{c}$, \\ C. Büttner ${ }^{b}$, A.A. Chilingarian ${ }^{d}$, K. Daumiller ${ }^{\text {a }}$, P. Doll ${ }^{b}$, \\ J. Engler ${ }^{\mathrm{b}}$, F. Fessler ${ }^{\mathrm{a}}$, H.J. Gils ${ }^{\mathrm{b}}$, R. Glasstetter ${ }^{\mathrm{a}}$, \\ R. Haeusler ${ }^{\text {a }}$, A. Haungs ${ }^{\text {b }}$, D. Heck ${ }^{\text {b }}$, J.R. Hörandel ${ }^{\text {a }}$, \\ A. Iwan ${ }^{a, 3}$, K.H. Kampert ${ }^{\text {a,b }}$, H.O. Klages ${ }^{b}$, G. Maier ${ }^{b}$, \\ H.J. Mathes ${ }^{\mathrm{b}}$, H.J. Mayer ${ }^{\mathrm{b}}$, J. Milke ${ }^{\mathrm{a}}$, M. Müller ${ }^{\mathrm{b}}$, \\ R. Obenland ${ }^{\mathrm{b}}$, J. Oehlschläger ${ }^{\mathrm{b}}$, S. Ostapchenko $^{\mathrm{a}, 4}{ }^{,}$ \\ M. Petcu ${ }^{c}$, H. Rebel $^{b}$, M. Risse $^{b}$, M. Roth $^{b}$, G. Schatz $^{b}$, \\ H. Schieler ${ }^{\mathrm{b}}$, J. Scholz ${ }^{\mathrm{b}}$, T. Thouw ${ }^{\mathrm{b}}$, H. Ulrich ${ }^{\mathrm{a}}$, J.H. Weber ${ }^{\mathrm{a}}$, \\ A. Weindl ${ }^{\mathrm{b}}$, J. Wentz ${ }^{\mathrm{b}}$, J. Wochele $^{\mathrm{b}}$, J. Zabierowski ${ }^{\mathrm{e}}$ \\ ${ }^{\mathrm{a}}$ University of Karlsruhe, Institut für Experimentelle Kernphysik, 76021 \\ Karlsruhe, Germany \\ ${ }^{\mathrm{b}}$ Forschungszentrum Karlsruhe, Institut für Kernphysik, 76021 Karlsruhe, \\ Germany \\ ${ }^{\mathrm{c}}$ National Institute of Physics and Nuclear Engineering, 7690 Bucharest, Romania \\ ${ }^{\mathrm{d}}$ Cosmic Ray Division, Yerevan Physics Institute, Yerevan 36, Armenia \\ e Soltan Institute for Nuclear Studies, 90950 Lodz, Poland
}

\begin{abstract}
Using the facilities of the KASCADE Central Detector EAS muon arrival time distributions, observed with reference to the arrival time of the first locally registered muon, and their correlations with other EAS observables have been experimentally

1 corresponding author: Florin.Badea@ik.fzk.de

2 on leave of absence from National Institute of Physics and Nuclear Engineering, Bucharest, Romania

3 on leave of absence from University of Lodz, Lodz, Poland

4 on leave of absence from Moscow State University, Moscow, Russia
\end{abstract}


investigated. The variation of adequately defined time parameters with the distance $R_{\mu}$ from the EAS axis has been measured. The experimental data enable a study of the sensitivity of such local arrival time distributions, which characterise the structure of the shower disc, to the mass composition of cosmic rays in the energy region around the knee. For that purpose, nonparametric multivariate evenby-event analyses have been performed for an estimate of the mass composition specified by three different mass groups, invoking detailed Monte Carlo simulations of the EAS development. It turns out that local muon arrival time distributions, without information on the curvature of the shower disc, display a minor sensitivity to the mass of the EAS inducing particle, at least for distances from the shower axis $R_{\mu}<100 \mathrm{~m}$. The measurements comprise a subset of all EAS events registered by KASCADE due to the observation conditions of the arrival time distributions, with a threshold of the muon energy $E_{t h}=2.4 \mathrm{GeV}$ and a minimum multiplicity $n_{t h}$ for being accepted in the observed data samples. This subset is sensitive to variations of the integral EAS muon energy spectrum. By studying the event acceptance in the registered samples on basis of Monte Carlo simulations a test of the consistency of the Monte Carlo simulations with the data is enabled, comparing the results inferred from observations at different $R_{\mu}$ and different $n_{t h}$ values. Within the present uncertainties the results of such a test show a remarkable agreement of the experimental findings with the Monte Carlo simulations, using the QGSJET model as generator of the high-energy hadronic interactions.

Key words: Extensive Air Showers, Arrival Time Distributions, Primary Mass Composition, Model Test

PACS: 98.70Sa, 96.40Pq

\section{Introduction}

The time delay of the particles inside the front of Extensive Air Showers (EAS) and the temporal structure of different EAS components are a subject of longstanding interest of cosmic ray research. In fact the first experimental studies have been performed in 1953 by Bassi, Clark and Rossi [1] and Jelly and Whitehouse [2], followed by many others [3]-[11]. The renewed interest arises from recent measurements using advanced detector facilities like the COVER PLASTEX detector within the GREX array [12]-[14], the EAS TOP array [15] or the facilities of the KASCADE experiment [16]. Investigations of the structure of the EAS disc by the KASCADE experiment are focused in particular on the muon component [17]-[21]. Due to the reduced influence of multiple Coulomb scattering of $\mathrm{GeV}$ muons and the absence of absorption, muons travel nearly in straight lines from the locus of production to the observing detector. Thus muon arrival time distributions, observed at large distances from the shower axis, are expected to map the longitudinal EAS development and to reflect the distribution of production heights via the 
time-of-flight of the muons. With some simplifications, using a triangulation procedure, the distribution of the production heights could be estimated from the time delay of the observed muons relative to the arrival time of the shower centre $[17,22,23]$. Basically the same information could be alternatively deduced from the distribution of angles of muon incidence relative to the shower axis [24]. Corresponding measurements are also a current subject of KASCADE investigations using the Muon Tracking Device [25]. Angle-of-incidence observations or the combination with arrival time measurements (as discussed with the term Time Track Complementary [22,26]) do not reveal additional basic information [23] though they provide an interesting practical alternative.

The basic sensitivity of muon arrival time distributions (whose phenomenological features seen with the KASCADE experiment are reported in Ref. [20]) to longitudinal EAS development and to the elongation rate [27-29] puts the question which particular parameters of the observed distributions provide useful signatures of the mass of the EAS primary and under which conditions they are helpful for the determination of the mass composition of the primary cosmic rays. The present paper addresses experimentally the question of the sensitivity to the primary mass on the basis of results from the KASCADE experiment, in particular of EAS event-by-event measurements of the temporal EAS structure (shower thickness) observed at relatively small distances to the shower axis $R_{\mu}<100 \mathrm{~m}$ for the primary energy range around the knee. Methodically non-parametric statistical analysis techniques of multivariate distributions [30] are applied for the mass classification of the observed EAS by their time parameters, derived from the muon arrival time distributions, and by their correlations with other EAS observables (like the shower size $N_{e}$ and the muon content $N_{\mu}$ ). For muon arrival time distributions the methods are outlined in Refs. [17,23]. These procedures avoid the bias of pre-chosen functional forms and compare to reference patterns, derived from extensive Monte Carlo simulations, by use of the EAS Monte Carlo simulation code CORSIKA [31], thus including all natural EAS fluctuations. By applying additional detailed detector simulations based on GEANT [32], the distortions arising from the experimental conditions [33] are taken into account.

The analysis of the EAS observations in terms of the mass of the primary introduces necessarily some model dependence by the high-energy hadronic interaction models invoked as generators of the Monte Carlo simulations. Therefore the results are subject of the uncertainities of the hadronic interaction model, used for the analysis. A possible way to evaluate the quality of a model is to derive the primary mass composition from the analysis of different observables and to consider the agreement or disagreement of the results. This idea has been worked out in a consistent and efficient manner [34] with an extended set of observables of the KASCADE experiment, showing that there exist systematic differences in the estimate of $\langle\ln A\rangle$, e.g., derived from different combinations of (correlated) observables [35], revealing the limitations of popular models like QGSJET [36], VENUS [37] and SIBYLL [38]. This indicates 
that improvements of the models are urgently needed on basis of experimental findings. The QGSJET model is actually considered to be that one of the best internal consistency [39]. Hence we refer furtheron to the QGSJET model.

The muon arrival time measurements imply a particular selection of EAS, with a distortion of the original mass composition in the subset of classified EAS. This is due to the energy threshold $E_{t h}(2.4 \mathrm{GeV}$ for KASCADE) of the muon detection and the condition of a minimum multiplicity $n_{t h}(=3$ muons detected by the KASCADE Central Detector) for reconstructing an arrival time distribution. The distortion is dependent on the particular values of $E_{t h}, n_{t h}$, on the mass $A$ of the primary and the muon content $N_{\mu}$, but also on the radial distance $R_{\mu}$ of the arrival time measuring detector from the shower axis. In order to restore the original mass composition corresponding efficiency (acceptance) correction factors have to be applied to the identified mass groups. These factors can be only determined by Monte Carlo simulations, studying the cuts implied by the experiment and their effect on the efficiency. An efficient and sensitive test of the interaction model used for the simulations and of the particle tracking procedures is enabled by applying the calculated correction factors to the results found at different $R_{\mu}$ and to look for the consistency of the resulting mass composition. Such a test of the Monte Carlo simulations and of the QGSJET model, especially in view of predictions of the muon energy spectra and muon densities and their fluctuations in eventby-event observations, is an important aspect of muon arrival time studies of this paper. The results add to the conclusions about the difficulties of the model in interpreting consistently muon density measurements with different muon energy thresholds [40].

\section{Muon arrival time measurements}

Measured arrival times $\tau_{1 \mu}<\tau_{2 \mu}<\tau_{3 \mu}<\ldots$ of muons registered by timing detectors at a distance $R_{\mu}$ from the shower axis, corrected by $\pm R_{\mu} \tan \theta / c$ ( $c$ - speed of light) in order to eliminate the distortions due to the shower inclination, refer to an experimentally provided zero-time. Depending on the choice of the kind of zero-time reference, there are two different types of muon arrival time distributions. By the use of the arrival time $\tau_{c}$ of the shower core as reference global arrival times are observed [33]:

$$
\Delta \tau_{i}^{g l o b}\left(R_{\mu}\right)=\tau_{i \mu}\left(R_{\mu}\right)-\tau_{c}
$$

e.g. for the foremost muon registered at $R_{\mu}$ :

$$
\Delta \tau_{1}^{g l o b}\left(R_{\mu}\right)=\tau_{1 \mu}\left(R_{\mu}\right)-\tau_{c}
$$


This type of time distributions informs about the curvature of the shower front as well as about the structure of the disc. The arrival time of the shower core is difficult to determine with sufficient precision. Therefore the analysis has been restricted to "local" times, which refer to the arrival of the foremost muon locally registered by the detector:

$$
\Delta \tau_{i}^{l o c}\left(R_{\mu}\right)=\tau_{i \mu}\left(R_{\mu}\right)-\tau_{1 \mu}\left(R_{\mu}\right) .
$$

(with omitting further the label loc), informing only about the thickness and the structure of the muon disc. Implications of observations of local muon arrival time distributions due to the fluctuations of the arrival of the first registered muon have been discussed in Ref. [33]. For event-by-event observations with a fluctuating number of muons (multiplicity), the individual relative arrival time distributions can be characterised by the mean values $\Delta \tau_{\text {mean }}$, and by various quantiles $\Delta \tau_{q}$, like the median $\Delta \tau_{0.50}$, the first quartile $\Delta \tau_{0.25}$ and the third quartile $\Delta \tau_{0.75}$ (for details of the definition see Refs. [19-21]). Their mean values and dispersion (standard deviations) represent the time profile of the EAS muon component.

KASCADE, whose layout is described in more detail in Ref. [16], is a multidetector system, installed in Forschungszentrum Karlsruhe (110 m a.s.l.), Germany, for the observation of extensive air showers in the primary energy range around the knee. One part is an array of 252 detector stations, distributed over an area of $200 \times 200 \mathrm{~m}^{2}$ on a grid of $13 \mathrm{~m}$ spacing for measuring the electron-photon component and the muon component with a threshold of $5 \mathrm{MeV}$ and $230 \mathrm{MeV}$, respectively and providing the basic information about arrival direction, core position, electron and muon sizes of the observed EAS. In particular, from the data of the field array the so-called truncated muon number $N_{\mu}^{t r}$, i.e. the muon density integrated between $40 \mathrm{~m}$ and $200 \mathrm{~m}$, is derived and used in the KASCADE case (between $10^{14}$ and $10^{16} \mathrm{eV}$ ) as an approximate mass independent energy identifier [41]. The location of the shower core can be determined (inside the fiducial area) with an uncertainty of less than $3 \mathrm{~m}$. The arrival direction of the shower is reconstructed with an uncertainty better than $0.5^{\circ}$. Details of the reconstruction procedures are described in Ref. [42]. As additional muon detector Limited Streamer Tubes tracking detectors have been installed in an underground tunnel for measurements of the lateral distribution $\left(E_{t h}=0.8 \mathrm{GeV}\right)$ and of muon angles-of-incidence distributions [25].

The muon arrival time measurements use, in particular, the facilities of the Central Detector [43] of KASCADE. It is basically an iron sampling calorimeter (with an area of $16 \times 20 \mathrm{~m}^{2}$, set up with liquid TMS and TMP ionisation chambers) for the identification and energy measurement of hadrons. In the basement of the set-up, below $3800 \mathrm{t}$ of iron and concrete, large-area position- 
sensitive multiwire proportional chambers (MWPC) [44] are operated for the identification of muons with $2.4 \mathrm{GeV}$ energy threshold. The performance of the detection system of the MWPC is improved by a layer of streamer tubes [45]. The trigger plane of the calorimeter is a system of 456 plastic scintillation detector elements $\left(47.5 \times 47.5 \times 3 \mathrm{~cm}^{3}\right.$ in size, each separated by a wavelength shifter) for providing a fast trigger signal (in addition to the trigger from the field array) for the MWPC, and for the timing measurements. Fast electronics [46] records low (muons) and high energy deposits (cascading hadrons). In order to remove the signals from cascading hadrons an upper limit of the energy deposit of $30 \mathrm{MeV}$ in each of the 456 scintillation counters is imposed. An amount of 24 millions of KASCADE events are used for the analysis [47]. Muon arrival time distributions have been reconstructed for muons with $E_{t h}=2.4 \mathrm{GeV}$ and for events with a number $n \geq 3$ of registered muons; after applying also some general cuts concerning the core position (within $100 \mathrm{~m}$ from the array centre), the angle of EAS incidence $\left(<40^{\circ}\right)$ and $\log _{10} N_{\mu}^{t r}(>3.6)$ the sample shrinked to approx. 240000 showers. The phenomenological features of the observed muon arrival time distributions have already been reported in Ref. [21], where further experimental details are communicated.

\section{EAS simulations}

The interpretation of the measured muon arrival time distributions and their correlations with other shower parameters need a-priori knowledge to be deduced from Monte Carlo simulations of the EAS development. The present analysis is based on simulations with the code CORSIKA (version 5.62) with a full and detailed simulation of the detector response. The simulations use the QGSJET (version 1998) model [36] as generator for high-energy interactions and GHEISHA [48] for interactions below $E_{l a b}=80 \mathrm{GeV}$. The electromagnetic part is treated by the EGS4 program [49]. Earth magnetic field, observation level and particle detection thresholds have been chosen in accordance to the experimental situation. The U.S. standard atmosphere [31] has been adopted for the simulations. The simulations have been performed for three different classes of primaries: protons $(\mathrm{H})$ for the light group, oxygen $(\mathrm{O})$ for the CNOgroup and iron (Fe) for the heavy group. The energy range covered by the simulations extends from $5.0 \cdot 10^{14} \mathrm{eV}$ to $3.06 \cdot 10^{16} \mathrm{eV}$ and the zenith angles comprise the range of $0^{\circ} \leq \theta \leq 40^{\circ}$. The centres of the simulated showers have been positioned inside a quadratic area of $210 \times 210 \mathrm{~m}^{2}$, slightly extend-

ing the KASCADE area. For each primary type approximately 90000 showers have been simulated, with decreasing numbers of shower events for higher primary energies and the larger zenith angles, due to restrictions in the computing time. Finally a sample for $0^{\circ} \leq \theta \leq 24^{\circ}$ (corresponding to a third of 

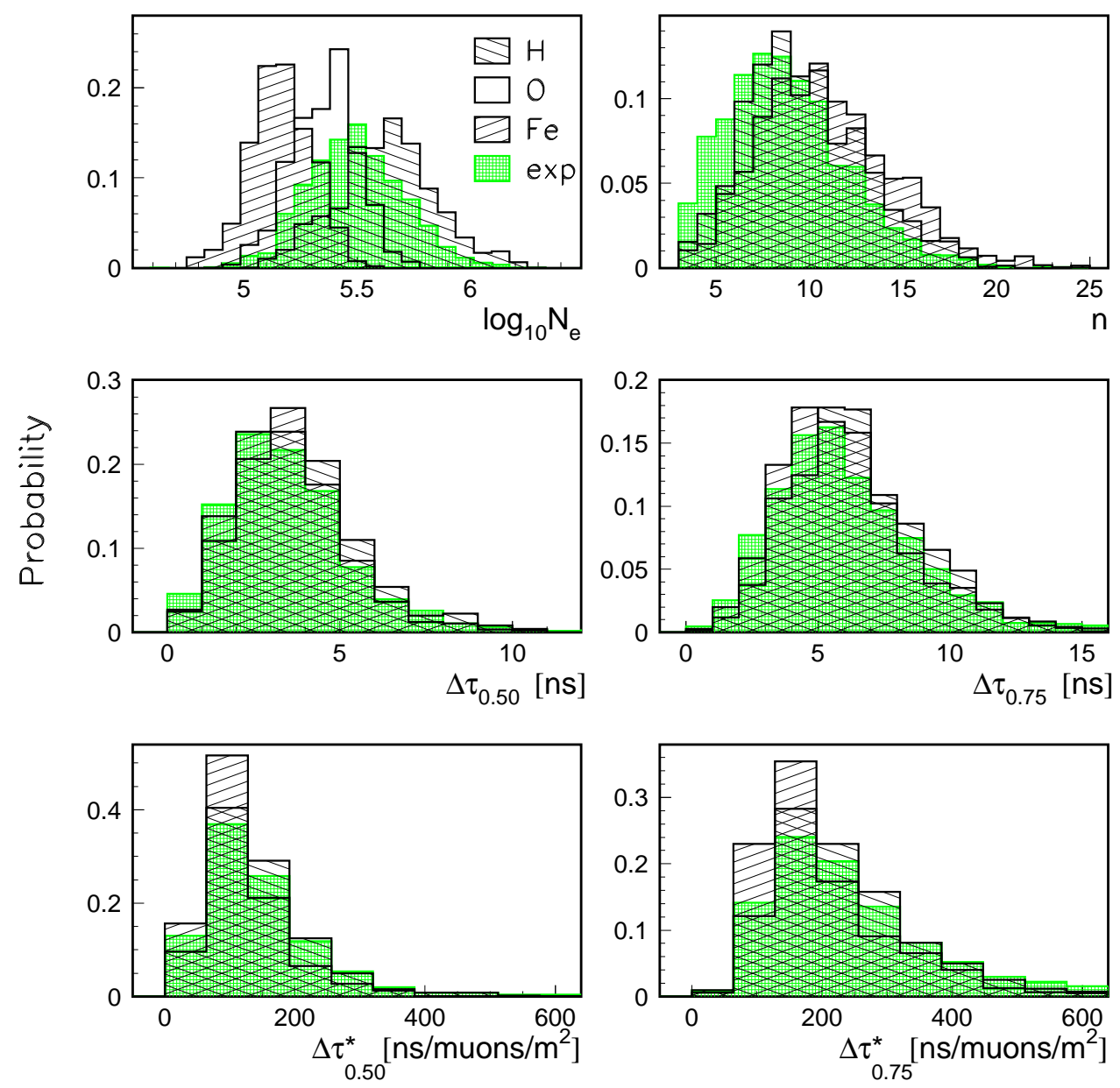

Fig. 1. The distribution of various shower observables of the sample, prepared by simulations for different types of primaries, compared with the distribution of the sample of experimental data for $4.05<\log _{10} N_{\mu}^{t r} \leq 4.28,80 \mathrm{~m}<R_{\mu} \leq 90 \mathrm{~m}$ and $0^{\circ} \leq \theta \leq 24^{\circ}$. The quantity $n$ is the multiplicity of muons $\left(E_{t h}=2.4 \mathrm{GeV}\right)$ detected with the MWPC setup of the Central Detector.

the considered $\sec \theta$ - range of $\left.\left(1, \sec 40^{\circ}\right)\right)$ was used in the analysis. The simulated primaries (separately for each of the cases $\mathrm{H}, \mathrm{O}, \mathrm{Fe}$ ) have been weighted according to:

$$
d N=\text { const. } \cdot E_{0}^{-\gamma} \cdot \sin \theta \cdot \cos \theta \cdot D_{\text {core }} \cdot d E_{0} \cdot d \theta \cdot d D_{\text {core }}
$$

where $d N$ is the number of primaries with energy between $E_{0}, E_{0}+d E_{0}$, zenith angle between $\theta, \theta+d \theta$, intersecting the plane of the detector array between $D_{\text {core }}, D_{\text {core }}+d D_{\text {core }}$. A fixed spectral index $\gamma=2.7$ over the whole primary energy range is adopted for all primaries $(\mathrm{H}, \mathrm{O}, \mathrm{Fe})$. More details about the simulations are given in Refs. [50] and [47]. As input for the further analysis of the muon arrival time distributions and their comparison with model predictions the following shower parameters and their correlations are regarded: 
- the shower size $N_{e}$;

- the truncated muon number $N_{\mu}^{t r}$;

- the multiplicity $n$ of muons $\left(E_{t h}=2.4 \mathrm{GeV}\right)$ detected in the facilities of the Central Detector;

- the quantiles $\Delta \tau_{q}$ of the local muon $\left(E_{t h}=2.4 \mathrm{GeV}\right)$ arrival time distributions at various distances $R_{\mu}$ from the shower centre;

- the reduced quantiles $\Delta \tau_{q}^{*}=\Delta \tau_{q} / \rho_{\mu}$ of the local muon arrival time distributions $\left(E_{t h}=2.4 \mathrm{GeV}\right)$, i.e. the quantiles divided by the density $\rho_{\mu}$, where $\rho_{\mu}$ is estimated from the observed multiplicity and the effective area of the muon detector set-up.

Fig. 1 displays frequency distributions of some shower parameters, prepared by the simulation for different primaries and compared to the actual experimental observations for particular $\log _{10} N_{\mu}^{t r}$ and $R_{\mu}$ bins. Each distribution is normalised (only for the presentation of $N_{e}$ the distribution of oxygen-induced EAS is additionally shown). There are some discrepancies in the multiplicity distribution between simulations and experimental observations. They may arise from an imperfect adoption of the primary energy spectrum and from efficiency effects not fully corrected for. Since they also affect the fluctuations of the local time parameters originating from the multiplicity dependence (discussed in detail in Ref. [33]), this analysis uses preferentially the reduced quantities $\Delta \tau_{q}^{*}$. The fluctuations largely cancel in the reduced parameters. Actually it has been argued that the reduced quantiles $\Delta \tau_{q}^{*}$ could exhibit enhanced mass sensitivity since they include approximately the $\Delta \tau_{q}\left(R_{\mu}\right)-\rho_{\mu}\left(R_{\mu}\right)$ correlation [51]. However this sensitivity is obscured by the limitations of the experimental response. CORSIKA simulations show $[23,51]$ that the age parameter is carrying relevant information about the longitudinal development. This aspect has not been worked out by the present analysis.

\section{Non-parametric multivariate analyses}

Non-parametric statistical methods are applied in studies of multidimensional distributions of observables allocating the single observed events to different classes (in our case to proton, oxygen, or iron primaries) by comparing the observed events with the simulated distributions without using a pre-chosen parameterisation. The methods of decision making and procedures of applications to cosmic ray data analyses are extensively described in Refs. [30,35] and outlined for analyses of muon arrival time distributions in Refs. [17,23]. The procedures take into account the effects of the EAS fluctuations in a quite natural way and are able to specify the uncertainties, by an estimate of the true-classification and misclassification probabilities. The classification probabilities are determined by the extent to which the likelihood functions of the individual classes, derived from the simulations, are overlapping. Ba- 
sically such pattern recognition methods, using trained neural networks or Bayes decision rules, depend on the hadronic model generating the reference patterns for the experimental observables. The concept of the present analysis is to determine in a first step the sensitivity of various observable correlations to the mass of the primary, by applying the one-leave-out test to the sample of distributions prepared by Monte Carlo simulations (QGSJET model). The one-leave-out test determines the probability that a multidimensional event, taken from the considered (simulated) distribution, will be correctly ("true") or incorrectly ("false") classified by the pattern recognition procedure (see appendix of Ref. [35]). With respect to the experimental data sample the studies are performed not only for six different $\log _{10} N_{\mu}^{t r}$ ranges between 3.60 and 5.00 (covering the knee-range) but also for four different $R_{\mu}$-ranges $(45-65 \mathrm{~m}$, 65-80 m, 80-90 m, 90-100 m). This allows to explore trends of the classification and misclassification probabilities and other quantities with varying $R_{\mu}$. Using the determined classification probabilities the mass composition is reconstructed from the data samples measured at different $R_{\mu}$-ranges. The resulting mass composition generally differs from the primary mass composition searched for, since the analysed data samples represent selections in the multidimensional space of all EAS observables. Hence efficiency correction factors have to be determined, whose application should lead to a primary mass composition consistent for all $R_{\mu}$-ranges. The determination of the efficiency correction factors invokes again Monte Carlo simulations, and the extent of agreement of the primary mass composition resulting from different $R_{\mu}$ ranges provides a test for the procedures and the generator of the Monte Carlo simulations.

\subsection{The classification and misclassification probabilities}

As example in Fig. 2 the true-classification and misclassification probabilities, inferred with the one-leave-out test from the simulation sample, are displayed for $\mathrm{H}, \mathrm{O}, \mathrm{Fe}$ primaries and for different combinations of observables of EAS events. The events have been registered with the conditions of muon arrival time measurements in a particular $\left\{\log _{10} N_{\mu}^{t r}, R_{\mu}\right\}$ - range. With little surprise it is noted that the mass discrimination is dominated by the $\left\{N_{\mu}^{t r}, N_{e}\right\}$ correlation (see Fig. 3), and it is also evident that local time parameters (shown for the median $\Delta \tau_{0.50}$ or the third quartile $\Delta \tau_{0.75}$ and their reduced values $\left.\Delta \tau_{0.50}^{*}, \Delta \tau_{0.75}^{*}\right)$ have minor influence on the discrimination. We emphasise that this statement holds for local arrival time distributions in the studied range of relatively small distances $R_{\mu}(<100 \mathrm{~m})$ from the shower centre. Theoretical studies $[47,51]$ indicate more pronounced differences in the temporal structure of the shower disc with increasing $R_{\mu}$ and $\log _{10} N_{\mu}^{t r}$. In contrast to results ignoring the detector response [51] the use of reduced parameters $\Delta \tau_{0.50}^{*}, \Delta \tau_{0.75}^{*}$ does not significantly improve the mass discrimination. Fig. 3 shows the true 


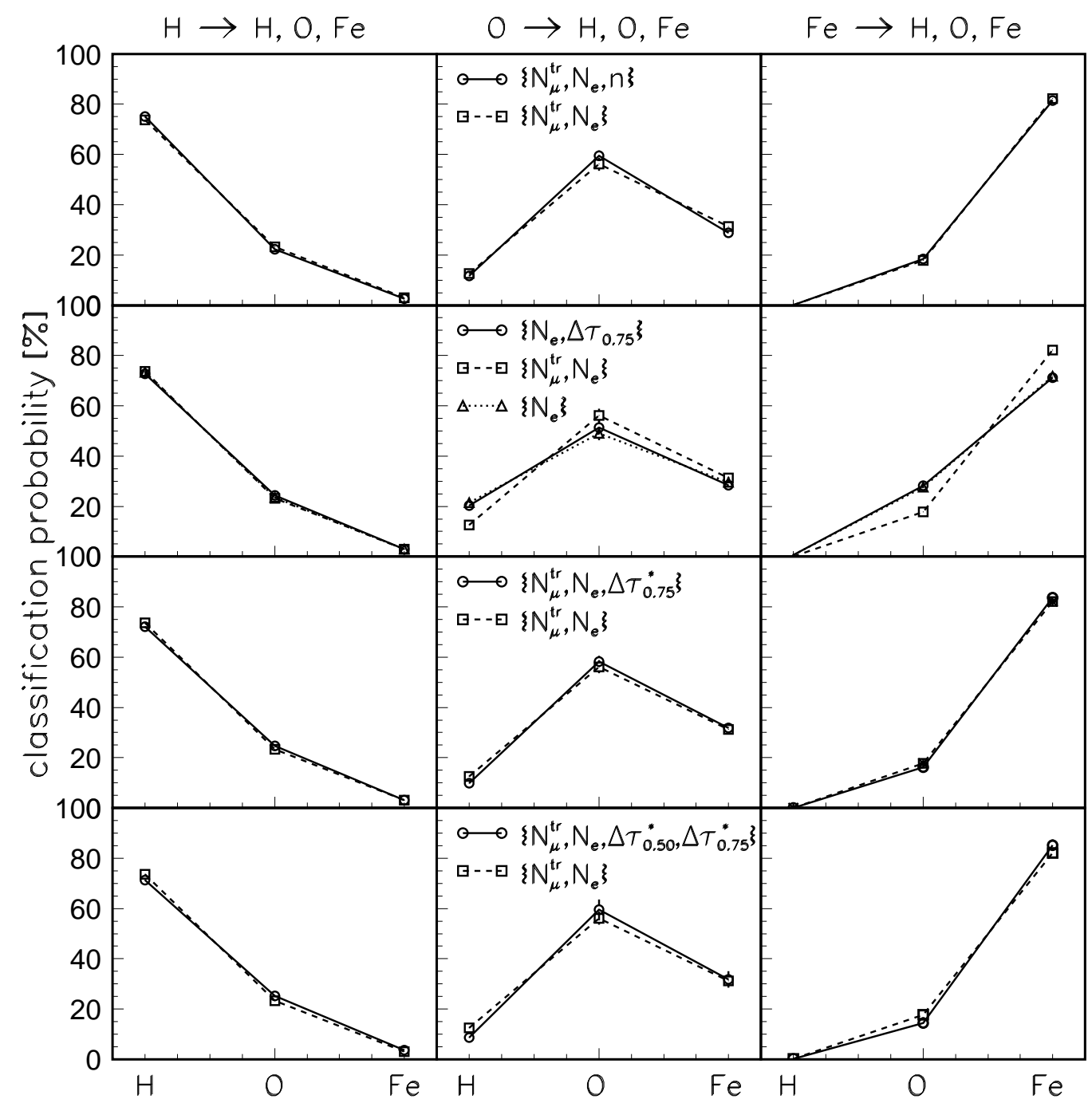

Fig. 2. Classification and misclassification probabilities determined by Bayes decision making for three different classes $(\mathrm{H}, \mathrm{O}, \mathrm{Fe})$ and for distribution of different types of correlated EAS observables with $3.83<\log _{10} N_{\mu}^{t r} \leq 4.05,80 \mathrm{~m}<R_{\mu} \leq$ $90 \mathrm{~m}, 0^{\circ} \leq \theta \leq 24^{\circ}$. The results of different observable combinations are connected by (full and dashed) lines.

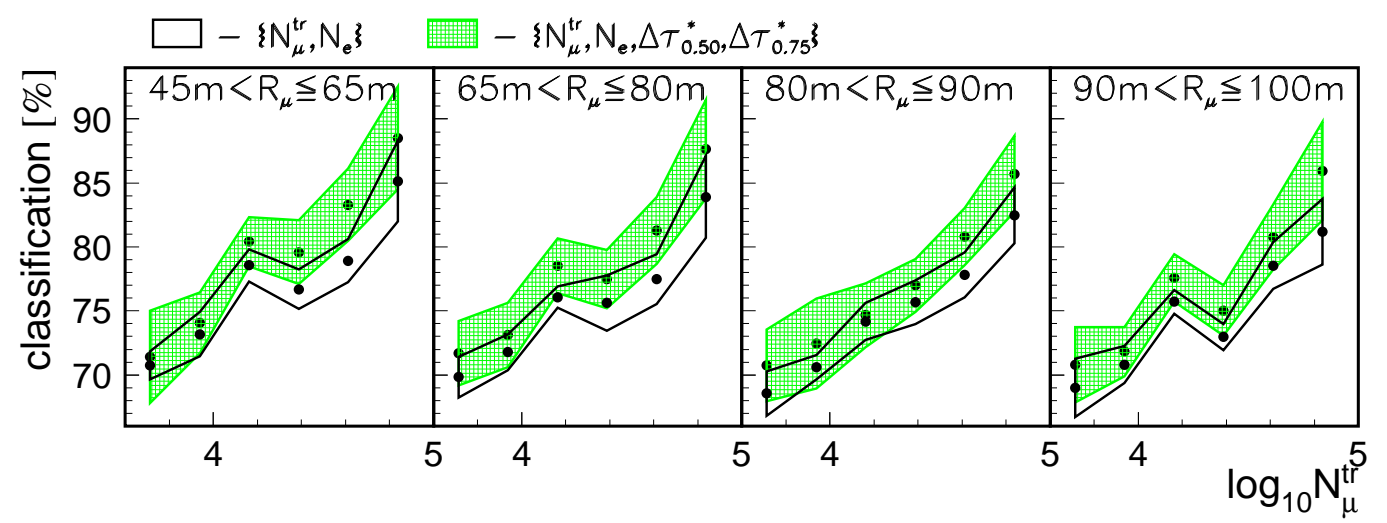

Fig. 3. The dependence of the averaged true-classification probability from $\left\{N_{\mu}^{t r}, N_{e}\right\}$ and $\left\{N_{\mu}^{t r}, N_{e}, \Delta \tau_{0.50}^{*}, \Delta \tau_{0.75}^{*}\right\}$ correlations $\left(0^{\circ} \leq \theta \leq 24^{\circ}\right)$. 
classification probabilities (and uncertainties) averaged over all classes with increasing $\log _{10} N_{\mu}^{t r}$ indicating a marginal systematic improvement if the time information is added. The uncertainties are determined by the bootstrap method [30], which basically consists of applying the classification procedure for a test sample several times, thus deriving an average value and the variance.

\subsection{Reconstruction of the mass composition from the observed data samples}

The true-classification $P_{i \rightarrow i}$ and misclassification $P_{i \rightarrow j}$ probabilities, deduced for all studied $\left\{\log _{10} N_{\mu}^{t r}, R_{\mu}\right\}$ ranges, are used for the reconstruction [23] of the mass composition of the samples of registered events by inverting the system of linear equations:

$$
\begin{aligned}
& n_{H}^{\prime}=n_{H} \cdot P_{H \rightarrow H}+n_{O} \cdot P_{O \rightarrow H}+n_{F e} \cdot P_{F e \rightarrow H} \\
& n_{O}^{\prime}=n_{H} \cdot P_{H \rightarrow O}+n_{O} \cdot P_{O \rightarrow O}+n_{F e} \cdot P_{F e \rightarrow O} \\
& n_{F e}^{\prime}=n_{H} \cdot P_{H \rightarrow F e}+n_{O} \cdot P_{O \rightarrow F e}+n_{F e} \cdot P_{F e \rightarrow F e}
\end{aligned}
$$

where $n_{H}, n_{O}, n_{F e}$ are the true numbers, defining the mass composition in the sample $N=n_{H}+n_{O}+n_{F e}$, getting altered to $n_{H}^{\prime}, n_{O}^{\prime}, n_{F e}^{\prime}$ because of the misclassifications. In Fig. 4 the reconstruction of an arbitrarily adopted mass

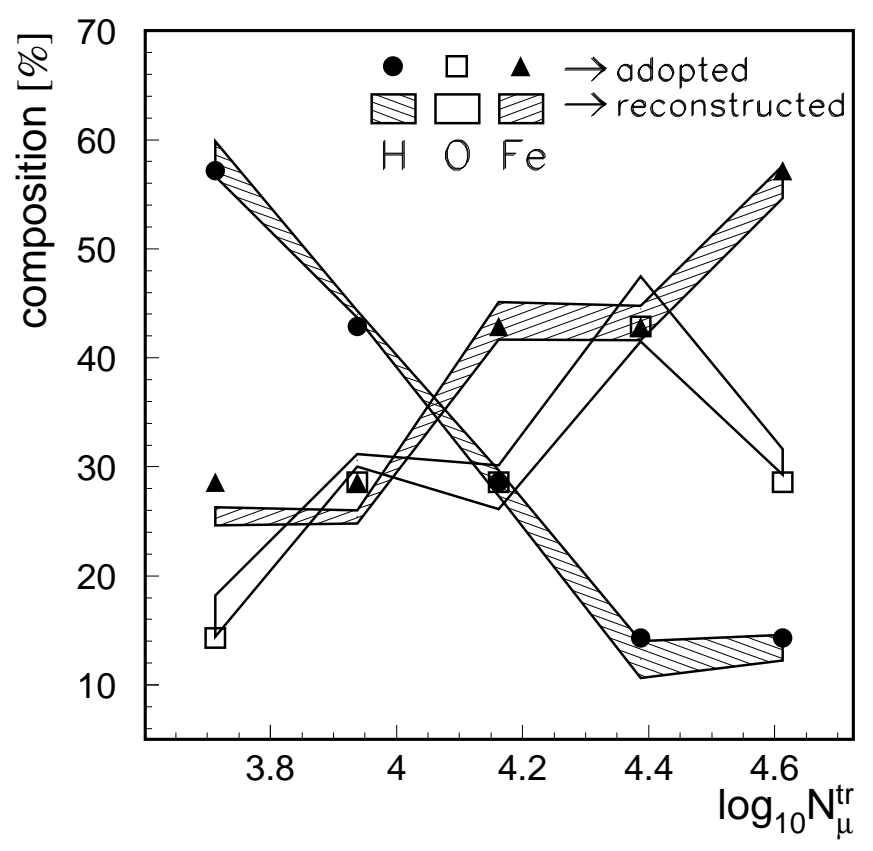

Fig. 4. Test of the reconstruction of an arbitrarily adopted mass composition of the event samples on basis of the $\left\{N_{\mu}^{t r}, N_{e}, \Delta \tau_{0.50}^{*}, \Delta \tau_{0.75}^{*}\right\}$ correlation, observed at $80 \mathrm{~m}$ $<R_{\mu} \leq 90 \mathrm{~m}, 0^{\circ} \leq \theta \leq 24^{\circ}$. 
composition of the event samples (displayed by the corresponding symbols), is shown with the resulting uncertainties as varying with $\log _{10} N_{\mu}^{t r}$. The result of the application of the reconstruction procedures to experimental samples measured with KASCADE, observed at different distances $R_{\mu}$ from the shower centre, are shown in the upper panels of Fig. 5. The uncertainties are influenced by the limited statistical accuracy of the data samples, in particular for the bins with larger $\log _{10} N_{\mu}^{t r}$ values i.e. primary energies. Nevertheless it is obvious that mass compositions of measured KASCADE samples differ for different $R_{\mu}$ bins, since the observation conditions lead to mass dependent differences in the observation efficiency at different $R_{\mu}$, leading to distortions of the deduced primary mass composition. This feature should be removed after applying a correct efficiency correction procedure. That necessarily implies again the use of the particular hadronic interaction model.

\subsection{Reconstruction of the primary mass composition}

Efficiency correction factors $\left(C_{H}, C_{O}, C_{F e}\right)$ have been calculated in order to adjust the mass composition of measured KASCADE samples $\left(P_{H}, P_{O}, P_{F e}\right)$ to the primary mass composition $\left(P_{H}^{*}, P_{O}^{*}, P_{F e}^{*}\right)$ according to the relation [47]:

$$
P_{H}^{*}: P_{O}^{*}: P_{F e}^{*}=\frac{P_{H}}{C_{H}}: \frac{P_{O}}{C_{O}}: \frac{P_{F e}}{C_{F e}} .
$$

As a first step, the simulated spectra given by eq. 4 have been normalised to the same value $(=1000)$ for each type of primaries $A(\mathrm{H}, \mathrm{O}, \mathrm{Fe})$, and for all simulated cases of $E_{0}, \theta$ and core position ranges. For a given primary $A$, the detected spectra will appear distorted at the ground level due to the absorption in the atmosphere and the selection cuts. Only a fraction of the original events are detected by the KASCADE detector and reconstructed successfully. The major influence on the values of the efficiency correction factors arises from the applied cuts on the reconstructed shower events. The correction factors depend on the $R_{\mu}, \theta$ and $\log _{10} N_{\mu}^{t r}$ ranges, on the multiplicity threshold for the $2.4 \mathrm{GeV}$ muons and, of course, on the primary type. For a given primary $A$, in a certain $\left\{R_{\mu}, R_{\mu}+\Delta R_{\mu} ; \theta, \theta+\Delta \theta ; \log _{10} N_{\mu}^{t r}, \log _{10} N_{\mu}^{t r}+\Delta \log _{10} N_{\mu}^{t r} ; n, n+\Delta n\right\}$ (multidimensional) bin, the correction factor $\left(C_{A}\right)$ is given by the sum of the weights $\left(w_{i}\right)$ of the $p$ simulated events which are accepted showers in the multidimensional bin:

$$
C_{A}=\sum_{i=1}^{p} w_{i}
$$

The weights $w_{i}$ are determined via the normalised mass spectra (eq. 4) which get filtered by the observation conditions. Considering the equivalent number 


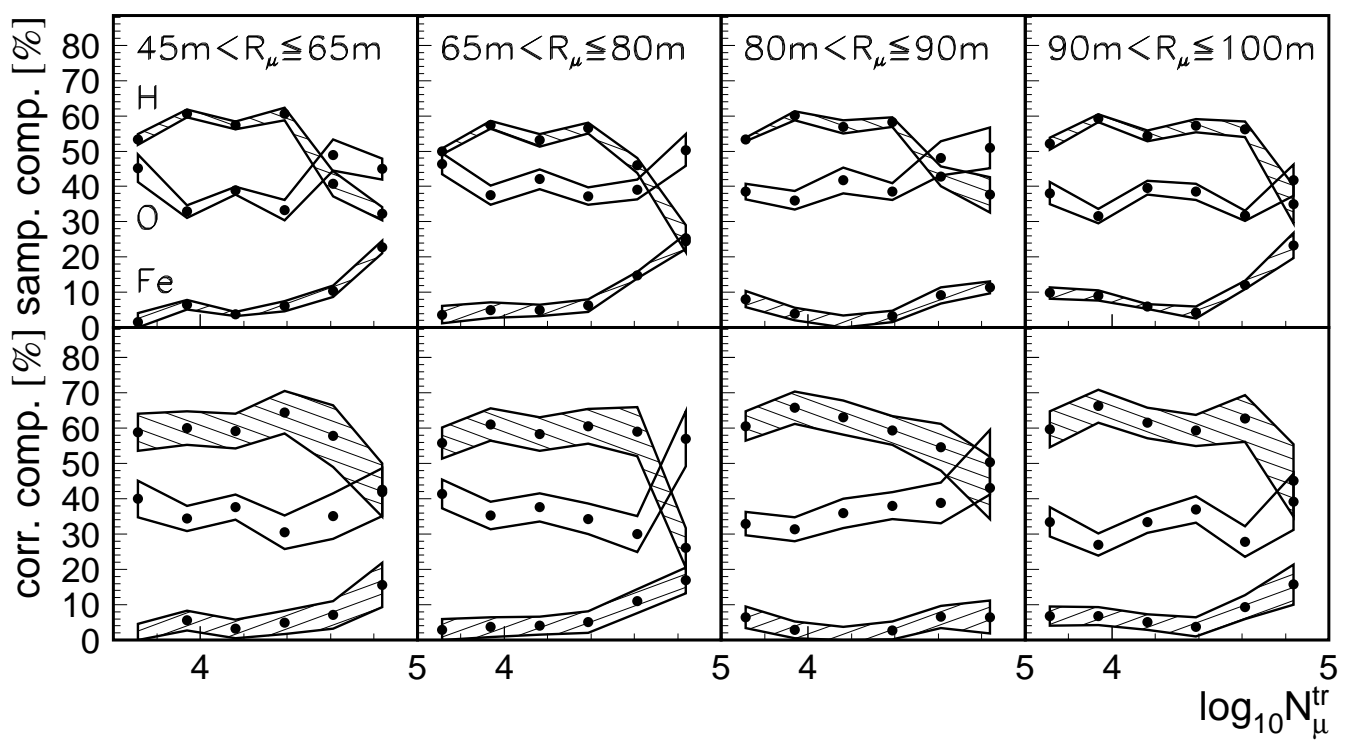

Fig. 5. Variation of the mass composition of measured KASCADE samples with $\log _{10} N_{\mu}^{t r}$ estimated from the $\left\{N_{\mu}^{t r}, N_{e}, \Delta \tau_{0.50}^{*}, \Delta \tau_{0.75}^{*}\right\}$ correlation and the primary mass composition i.e. before (samp. comp.) and after (corr. comp.) efficiency correction shown for different ranges $R_{\mu}$ from the shower centre, for the zenith angle range $0^{\circ} \leq \theta \leq 24^{\circ}$.

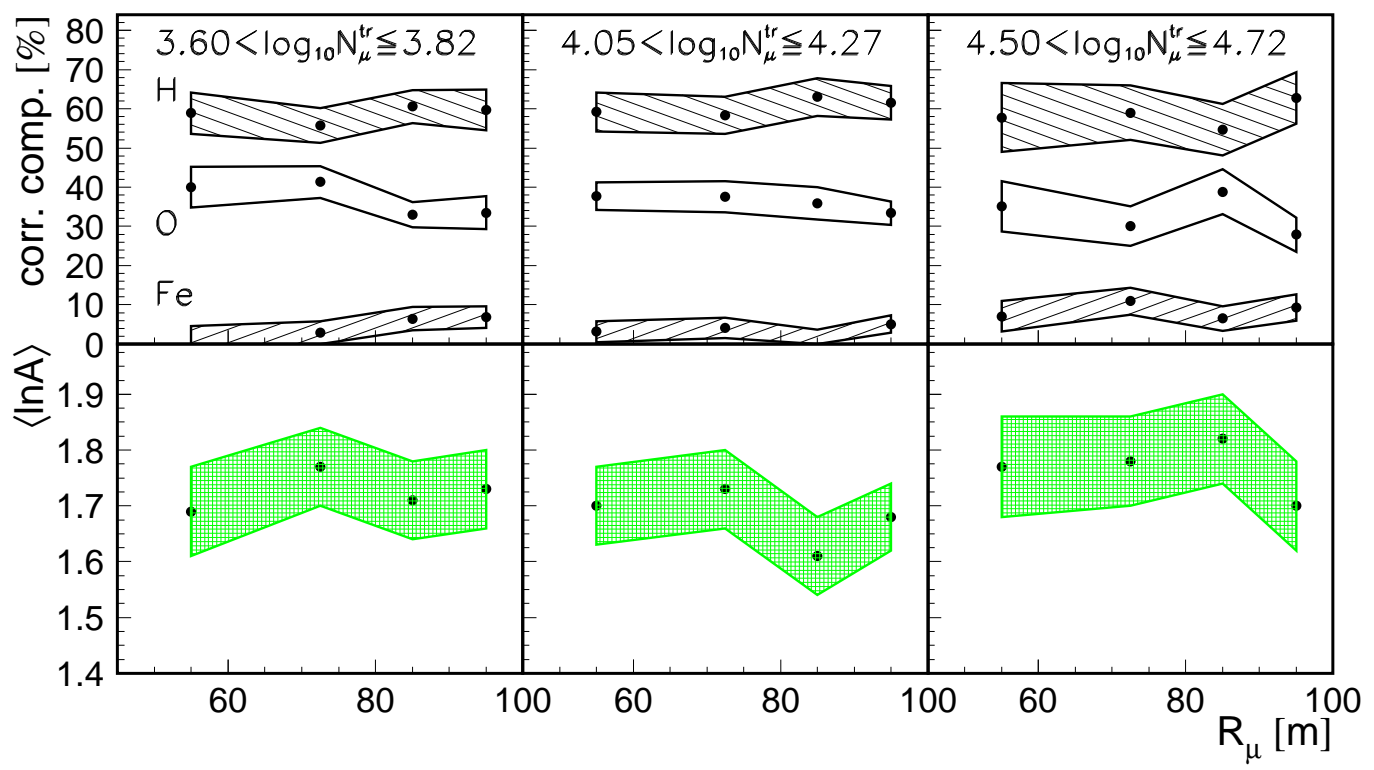

Fig. 6. Variation of the efficiency corrected mass composition and of $\langle\ln A\rangle$ with $R_{\mu}$ for various $\log _{10} N_{\mu}^{t r}$ ranges; $0^{\circ} \leq \theta \leq 24^{\circ}$.

of events $q[52]$ given by: 


$$
q=\frac{\left(\sum_{i=1}^{p} w_{i}\right)^{2}}{\sum_{i=1}^{p} w_{i}^{2}}
$$

the statistical uncertainty (error of the mean value) has been calculated for $C_{A}$ by:

$$
\delta C_{A}=C_{A} \cdot \frac{\sqrt{q}}{q}=\frac{C_{A}}{\sqrt{q}} .
$$

Fig. 5 displays for different $R_{\mu}$-ranges the $\log _{10} N_{\mu}^{t r}$-variation of the mass composition of the measured KASCADE samples extracted from the $\left\{N_{\mu}^{t r}, N_{e}\right.$, $\left.\Delta \tau_{0.50}^{*}, \Delta \tau_{0.75}^{*}\right\}$ correlation and the primary mass composition resulting after the correction concerning the biased acceptance by the specific observational conditions. In the lower part of Fig. 6 the results are shown as variation of $\langle\ln A\rangle$ with $R_{\mu}$ for different $\log _{10} N_{\mu}^{t r}$ ranges. The uncertainties of the correc-

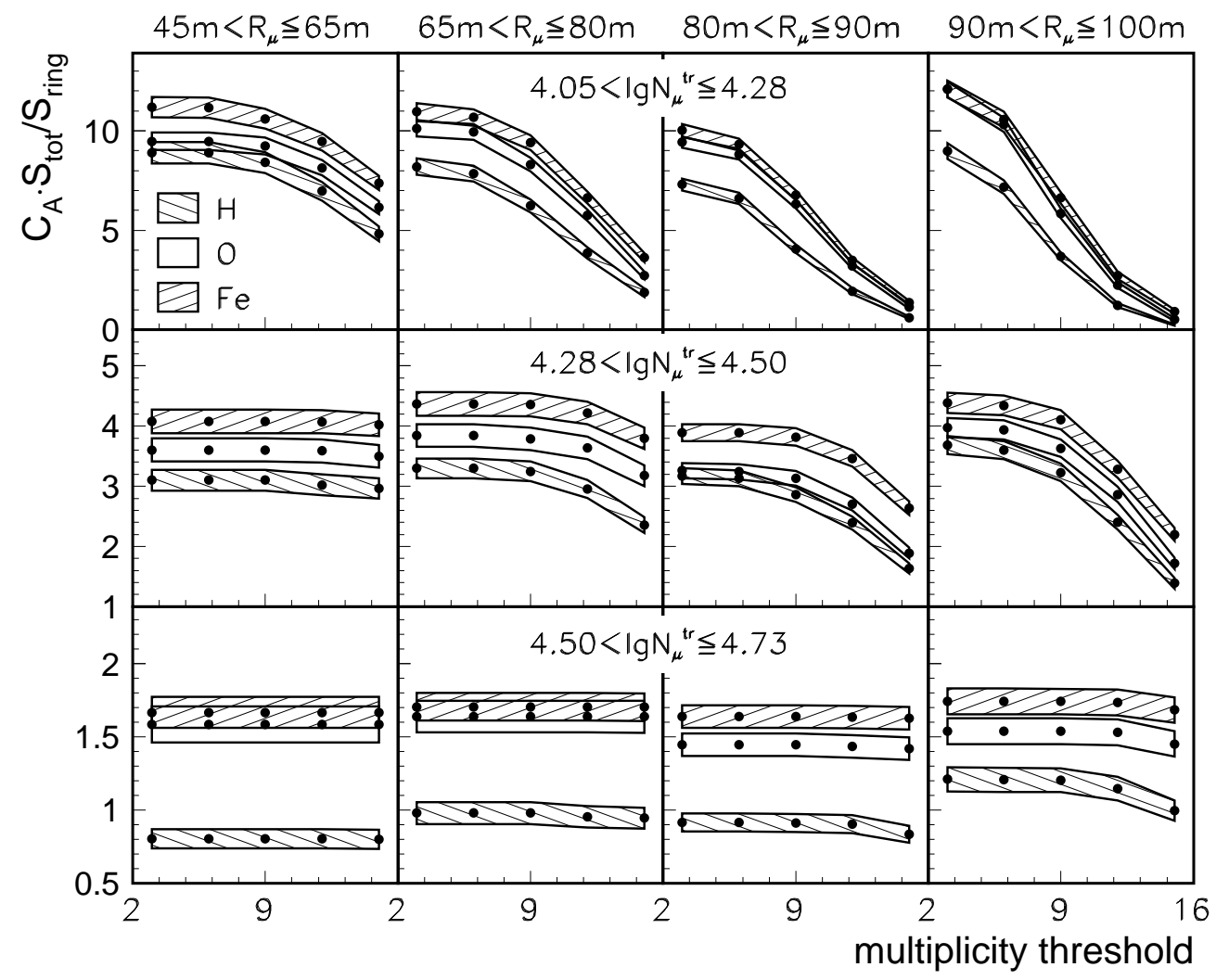

Fig. 7. Variation of the calculated acceptance correction factors with $n_{t h}$ for four $R_{\mu}$ ranges and three $\log _{10} N_{\mu}^{t r}$ ranges in the zenithal angle range of $0^{\circ} \leq \theta \leq 24^{\circ}$. The factor $S_{t o t} / S_{\text {ring }}\left(S_{\text {tot }}=210 \times 210 \mathrm{~m}^{2}\right)$ accounts for the different geometrical areas for different $R_{\mu}$ ranges. 
tion factors (eq. 9) have been included by propagation via eq. 6. It should be stressed that the displayed error bands do not include the uncertainty of the adopted spectral indices $\gamma$. Within the overall uncertainties the results obtained for different $R_{\mu}$ ranges are in fair agreement. This can be considered as a proof of the consistency of the Monte Carlo simulations invoked for the analysis of the data. The correction factors $C_{A}$ give the fraction of the primary energy spectrum of the considered primary mass which contributes to the $\left\{N_{\mu}^{t r}, N_{e}, \ldots\right\}$ sample observed with conditions specified by the energy threshold of the registered muons $E_{t h}$, the distance from the shower centre and the multiplicity threshold $n_{t h}$. In order to give an impression on these factors, they are shown for various cases with their variation with $n_{t h}$ in Fig. 7 . The variation of $C_{A}$ is more pronounced at smaller $N_{\mu}^{t r}$ values (i.e. primary energies), and as expected from the muon lateral distributions the acceptance of Fe events in the observation sample is higher than for proton events.

\section{Test of the consistency of Monte Carlo simulations}

As emphasised above, the event samples registered for these measurements are particular selections of all EAS events, determined by the observation conditions, especially by the energy threshold of the detected muons and the multiplicity threshold. Thus the event selections are affected by the lateral variation and mass dependence of the muon energy spectrum of EAS. The mass composition of these samples, as inferred from the correlations of the observed EAS observables, are altered as compared to the primary composition, in a way varying with the distance $R_{\mu}$ from shower centre. The acceptance or efficiency factors $C_{A}$ for correcting this effect depend on the Monte Carlo simulations and their ingredients like the model descriptions of the hadronic interaction. Since the measurements are performed at different distances from the EAS centre, the variation of the results after applying the $C_{A}$ factors implies a consistency test of the Monte Carlo simulations since the final result should be independent from $R_{\mu}$ within the given uncertainties. This test can be refined and be more stringent when not only the variation with $R_{\mu}$, but also the variation with the multiplicity threshold $n_{t h}$ is scrutinised. In Fig. 8 the results of such a refined test are shown, based on the dominant $\left\{N_{\mu}^{t r}, N_{e}\right\}$ correlation within the observed samples. Observables describing the (local) EAS time structure (which are shown to be of minor sensitivity) have not been included in the classification procedure though their measurement defines the test sample. The results have been obtained by repeating the full data analysis, described in the previous sections, for different multiplicity thresholds. In view of the rather specific observation conditions, sensitive to variations of the EAS muon energy spectrum, we consider the results, even if display-

ing larger uncertainties, as a remarkable confirmation of the consistency of 


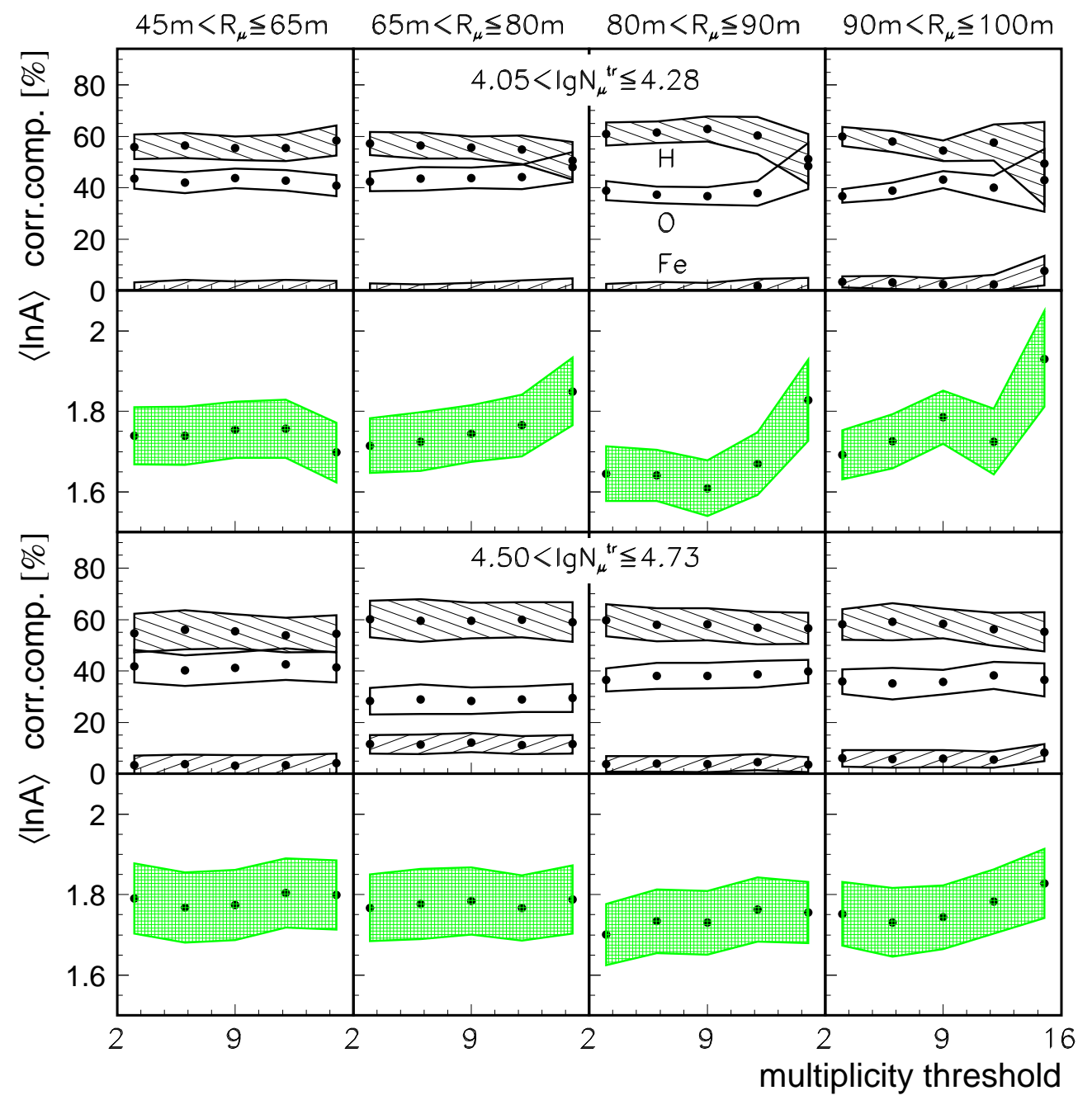

Fig. 8. Variation of the deduced primary mass composition with the multiplicity threshold $n_{t h}$ using the $\left\{N_{\mu}^{t r}, N_{e}\right\}$ correlation for the non-parametric classification procedure of the events observed at different distances from the shower axis. Shown are results for two different $\log _{10} N_{\mu}^{t r}$ ranges (corresponding to primary energies below and above the knee in the primary cosmic ray spectrum).

the Monte Carlo simulations performed with the CORSIKA code using the QGSJET model. The test could be improved when samples resulting from specific cuts would be analysed by simultaneously classifying the primary energy and the mass, using efficient pattern recognition methods as applied in other KASCADE studies [34,35]. The mass composition resulting from the present studies is in fair agreement with the results of those investigations. In particular it corroborates the finding of an increase of $\langle\ln A\rangle$ beyond the knee, i.e. for $\log _{10} N_{\mu}^{t r}>$ ca. 4.2 . 


\section{Conclusions}

The present investigation considers experimental data of the KASCADE experiment which are based on observations of local muon arrival time distributions, representing the variation of the EAS disc thickness with the distance from the shower axis. The first focus of the studies is the question to which extent these time quantities and their correlations with other EAS observables can help to improve the mass discrimination in the event samples observed with realistic experimental, i.e. with KASCADE conditions. Methodically the event-by-event analyses apply a non-parametric approach with reference patterns provided by CORSIKA simulations of the EAS development, using as generators the QGSJET model for the high energy interaction part and GHEISHA for the low energy part. It turns out that similarly to other EAS observables, the local time quantities provide only a marginal contribution to the mass discrimination as compared to the dominant $\left\{N_{\mu}^{t r}, N_{e}\right\}$ correlation, at least in the relatively limited range of $R_{\mu}<100 \mathrm{~m}$. It should be emphasised that this statement does by no means disqualify the sensitivity of global muon arrival time distributions measured relative to the arrival time of the shower centre. In fact, studies including the curvature of the shower front indicate a considerable enhancement of the mass discrimination, in particular at larger distances from the shower core and higher primary energies $[47,51]$.

The event sample collected with the observation of muon arrival time distributions in the KASCADE experiment is a subset of all EAS events measured with $100 \%$ efficiency for $\log _{10} N_{\mu}^{t r} \geq$ approx. 3.6. As consequence of the muon energy threshold $E_{t h}=2.4 \mathrm{GeV}$ and the condition of a registered multiplicity $n \geq 3$ the original primary mass composition in the registered samples gets distorted in a way which is sensitive to lateral variations of the integral EAS muon energy spectrum. In order to determine the fraction of all shower events which are accepted in the specific subset, specified by the measuring conditions, Monte Carlo simulations have to be invoked for the calculation of corresponding acceptance or efficiency factors. The variation of such corrections with the distance of observation $R_{\mu}$ from the shower axis and with the multiplicity threshold $n_{t h}$ provides the possibility to test the consistency of the Monte Carlo simulations with the data. Even admitting the large uncertainties due to the limited number of events observed and simulated, our results indicate a remarkable consistency of the performed Monte Carlo simulations using the QGSJET model as generator.

\section{Acknowledgements}

We acknowledge various useful discussions with Prof. Dr. M. Petrovici about the aspects of the results elaborated in this paper. The authors would like to 
thank the members of the engineering and technical staff of the KASCADE collaboration who considerably contributed with enthusiasm and expert engagement to the success of the reported measurements. The work has been supported by the Ministry of Research of the Federal Republic of Germany. The Romanian Ministry of Education and Research provided a grant for supporting the collaborating group of the National Institute for Physics and $\mathrm{Nu}-$ clear Engineering of Bucharest. The Polish collaborating group of the Cosmic Ray Division of the Soltan Institute for Nuclear Studies, Lodz, is supported by the Polish State Committee for Scientific Research (grant No. 5 P03B 133 20). The KASCADE collaboration work is embedded in the frame of scientifictechnical co-operation (WTZ) projects between Germany and Romania (ROM 99/005), Poland (POL 99/005) and Armenia (ARM 98/002).

\section{References}

[1] P. Basi, G. Clark and B. Rossi, Phys. Rev. 92 (1953) 441

[2] J.V. Jelly and W.J. Whitehouse, Proc. Phys. Soc. (London) A66 (1953) 454

[3] J. Linsley, L. Scarsi, B. Rossi, Phys. Rev. Lett. 6 (1961) 485

[4] J. Linsley and L. Scarsi, Phys. Rev. 128 (1962) 2384

[5] C.P. Woidneck and E. Böhm, J. Phys. A: Math. Gen. 8 (1975) 997

[6] A.A. Watson and J.G. Wilson, J. Phys. A: Math. Gen. 7 (1976) 1199

[7] J. Linsley, J. Phys. G: Nucl. Part. Phys. 12 (1986) 51

[8] A.J. Baxter, A.A. Watson and J.G. Wilson, Canadian J. Phys. 46 (1968) S9

[9] P.R. Blake et al., J. Phys G: Nucl. Part. Phys 16 (1990) 755

[10] G.B. Khristiansen et al., Proc. 21th ICRC, Adelaide, Australia 1990, vol. 9, p. 150

[11] J.A. Goodman et al., Phys. Rev. Lett. 42 (1979) 854.

[12] G. Agnetta et al., Astrop. Phys. 6 (1997) 301

[13] M. Ambrosio et al., Astrop. Phys. 7 (1997) 329

[14] M. Ambrosio et al., Astrop. Phys. 11 (1999) 427

[15] EAS-TOP collaboration, Nucl. Instr. and Meth. A 336 (1993) 310

[16] H.O. Klages et al. - KASCADE Collaboration, Nucl. Phys. B., Proc. Supl. 52B (1997) 92; H. Rebel and the KASCADE Collaboration, Proc. VII. Intern. Symposium and Very High Energy Cosmic-Ray-Interactions, Ann Arbor (Michigan), June 21-27, 1992 - AIP Conf. Proc. 276, ed. L. Jones

[17] H. Rebel et al., Phys. G: Nucl. Part. Phys. 21 (1995) 451; H. Rebel, Proceedings XV Cracow Summer School of Cosmology The Cosmic Ray Mass Composition, Lodz - Poland, 15-19 July, 1996, ed. W. Tkaczyk, p. 91

[18] M. Föller, U. Raidt et al. - KASCADE Collaboration Proc. 25th ICRC, Durban, South Africa, 1997, vol. 6, p. 149 
[19] I.M. Brancus et al. - KASCADE Collaboration, FZKA- Report 6151, Forschungszentrum Karlsruhe 1998

[20] I.M. Brancus et al. - KASCADE Collaboration: Proc. 26th ICRC, Salt Lake City, USA, Aug. 1999, eds. D.Kieda et al., vol.1, p.345

[21] T. Antoni et al. - KASCADE Collaboration, Astrop. Phys. 15 (2001) 149

[22] T.V. Danilova et al., Phys. G: Nucl. Part. Phys. 20 (1995) 961

[23] I.M. Brancus et al., Astrop. Phys. 7 (1997) 343

[24] K. Bernlöhr, Astrop. Phys. 5 (1996) 139

[25] J. Zabierowski et al. - KASCADE Collaboration, Proc. 27th ICRC Hamburg, Germany, 2001, vol. 2, p. 810; P. Doll et al., Nucl. Instr. and Meth. A to be published

[26] M. Ambrosio et al., J. Phys. G: Nucl. Part. Phys.23 (1997) 219

[27] J. Linsley and A.A. Watson, Phys. Rev. Lett. 46 (1981) 459

[28] R. Walker and A.A. Watson, J. Phys. G: Nucl. Phys. 7 (1981) 1297

[29] A.F. Badea et al., Astrop. Phys. 15 (2001) 19

[30] A.A. Chilingarian, Comp. Phys. Comm. 54 (1989) 381; A.A. Chilingarian and G.Z.Zazian, Nuovo Cim. 14 (1991) 355; ANI reference manual, 1999 (unpublished)

[31] D. Heck et al., FZKA-Report 6019, Forschungszentrum Karlsruhe (1998)

[32] R. Brun et al., GEANT 3, Report CERN DD/EE/84-1(1987)

[33] R. Haeusler et al., Astrop. Phys. (2002), in press

[34] M. Roth et al. - KASCADE Collaboration, Proc. 27th ICRC, Hamburg, Germany, 2001, vol. 1, p. 88

[35] T. Antoni et al. - KASCADE Collaboration, Astrop. Phys. 16 (2002) 245

[36] N.N. Kalmykov, S. Ostapchenko and A.I. Pavlov, Nucl. Phys. B. Proc. Suppl. 52B (1997) 17

[37] K. Werner, Phys. Rep. 232 (1993) 87

[38] R.S. Fletcher et al., Phys. Rev. D50 (1994) 5710

[39] T. Antoni et al. - KASCADE Collaboration, J. Phys. G: Nucl. Part. Phys. 25 (1999) 2161

[40] T. Antoni et al. - KASCADE Collaboration, Astrop. Phys. 16 (2002) 373

[41] J.H. Weber et al. - KASCADE Collaboration, Proc. 25th ICRC Durban, South Africa, 1997, vol. 6, p.153

[42] T. Antoni et al. - KASCADE Collaboration, Astrop. Phys. 14 (2001) 245

[43] J. Engler et al., Nucl. Instr. and Meth. A 427 (1999) 528

[44] H. Bozdog et al., Nucl. Instr. and Meth. A 465 (2001) 45

[45] T. Antoni et al. - KASCADE Collaboration, Proc. 27th ICRC Hamburg, Germany, 2001, vol. 2 p. 806

[46] M. Brendle and U. Raidt, Nucl. Instr. and Meth. A 412 (1998) 420 
[47] A.F. Badea, FZKA-Report 6579, Forschungszentrum Karlsruhe (2001)

[48] H. Fesefeldt, PITHA- 85/02, RWTH Aachen (1985)

[49] W.R. Nelson, H. Hirayama and D.W.O. Rogers, Report SLAC 265, Stanford Linear Accelerator Center (1985)

[50] R. Haeusler, FZKA-Report 6520, Forschungszentrum Karlsruhe (2000); in German

[51] I.M. Brancus et al., Analysis of muon arrival time distributions of simulated extensive air showers using advanced statistical procedures in view of observations with KASCADE and KASCADE Grande, Report WP17 IDRANAP 04-01/2001, IFIN-HH Bucharest, 2001

[52] HBOOK 4.21, Reference Manual, CERN (1993), p. 93 